Article

\title{
The Extremal Cacti on Multiplicative Degree-Kirchhoff Index
}

\author{
Fangguo $\mathrm{He}^{1}{ }^{1(1)}$ and Zhongxun $\mathrm{Zhu}^{2, *}$ \\ 1 College of Mathematics and Physics, Huanggang Normal University, Huanggang 438000, China; \\ hfg0118@126.com \\ 2 College of Mathematics and Statistics, South Central University for Nationalities, Wuhan 430074, China \\ * Correspondence: zzxun73@mail.scuec.edu.cn
}

Received: 22 November 2018; Accepted: 10 January 2019; Published: 15 January 2019

\begin{abstract}
For a graph $G$, the resistance distance $r_{G}(x, y)$ is defined to be the effective resistance between vertices $x$ and $y$, the multiplicative degree-Kirchhoff index $R^{*}(G)=\sum_{\{x, y\} \subset V(G)} d_{G}(x) d_{G}(y) r_{G}(x, y)$, where $d_{G}(x)$ is the degree of vertex $x$, and $V(G)$ denotes the vertex set of $G$. L. Feng et al. obtained the element in $\operatorname{Cact}(n ; t)$ with first-minimum multiplicative degree-Kirchhoff index. In this paper, we first give some transformations on $R^{*}(G)$, and then, by these transformations, the second-minimum multiplicative degree-Kirchhoff index and the corresponding extremal graph are determined, respectively.
\end{abstract}

Keywords: resistance distance; multiplicative degree-Kirchhoff index; cactus

\section{Introduction}

Throughout this paper, we consider finite, undirected simple graphs. Let $G=(V(G), E(G))$ be a graph with vertex set $V(G)$ (or $V$ ) and edge set $E(G)$. For a graph $G$, the distance between vertices $x$ and $y$, denoted by $d_{G}(x, y)$, is the length of a shortest path between them.

For distance, Harold Wiener in 1947 defined a famous index $W(G)$ [1], named Wiener index, where $W(G)=\sum_{x, y \in V} d_{G}(x, y)$. It is the earliest and one of the most thoroughly studied distance-based graph invariants. Later, Dobrynin and Kochetova [2] gave a modified version of the Wiener index $D^{+}(G)=\sum_{x, y \in V}\left(d_{G}(x)+d_{G}(y)\right) d_{G}(x, y)$. It is called degree distance and has attracted much attention (see [3-6]). For a graph $G$, the degree distance $D^{+}(G)$ is the essential part of the molecular topological index $\operatorname{MTI}(G)$ introduced by Schultz [7], which is defined as $\operatorname{MTI}(G)=\sum_{x \in V} d_{G}^{2}(x)+D^{+}(G)$, where $\sum_{x \in V} d_{G}^{2}(x)$ is the well-known first Zagreb index [8]. Klein et al. [9] discovered the relation between degree distance and Wiener index for a tree $G$ on $n$ vertices:

$$
D^{+}(G)=4 W(G)-n(n-1) .
$$

The Gutman index of a connected graph $G$ is defined as $D^{*}(G)=\sum_{x, y \in V} d_{G}(x) d_{G}(y) d_{G}(x, y)$. It was introduced in [10] and has been studied extensively (see, e.g., [11,12]). For a tree $G$ on $n$ vertices, Gutman [10] showed that

$$
D^{*}(G)=4 W(G)-(n-1)(2 n-1)
$$

In 1993, Klein and Randić [13] introduced a distance function named resistance distance on a graph. They viewed a graph $G$ as an electrical network such that each edge of $G$ is assumed to be a unit resistor, and the resistance distance between the vertices $x$ and $y$ of the graph $G$, denoted by $r_{G}(x, y)$, is then defined to be the effective resistance between the vertices $x$ and $y$ in $G$. The Kirchhoff index $K f(G)$ of $G$ is defined as 


$$
K f(G)=\sum_{x, y \in V} r_{G}(x, y)
$$

The index has been widely studied in mathematical, physical and chemical aspects; for details on the Kirchhoff index, the readers are referred to [14-18]. In 1996, Gutman and Mohar [19] obtained the result by which a relationship is established between the Kirchhoff index and the Laplacian spectrum: $K f(G)=n \sum_{i=1}^{n-1} \frac{1}{\mu_{i}}$, where $\mu_{1} \geq \mu_{2} \geq \ldots \geq \mu_{n}=0$ are the eigenvalues of the Laplacian matrix of a connected graph $G$ with $n$ vertices.

Similarly, if the distance is replaced by resistance distance in the expression for the degree distance and Gutman index, respectively, then one arrives at the following indices

$$
\begin{aligned}
R^{+}(G) & =\sum_{x, y \in V}\left(d_{G}(x)+d_{G}(y)\right) r_{G}(x, y), \\
R^{*}(G) & =\sum_{x, y \in V} d_{G}(x) d_{G}(y) r_{G}(x, y) .
\end{aligned}
$$

$R^{+}(G)$ and $R^{*}(G)$ are called the additive degree-Kirchhoff index and multiplicative degreeKirchhoff index, respectively, and were introduced by Gutman et al. [20] and Chen et al. [21], respectively. The indices have been well studied in both mathematical and chemical literature. In [22] some properties of $R^{+}(G)$ are determined and the extremal graph of cacti with minimum $R^{+}$-value characterized. Bianchi et al. [23] studied some upper and lower bounds for $G^{+}(G)$ whose expressions do not depend on the resistance distances. Feng et al. [24] characterized $n$-vertex unicyclic graphs having maximum, second maximum, minimum, and second minimum multiplicative degree-Kirchhoff index. Palacios [25] studied some interplay of the three Kirchhoff indices and found lower and upper bounds for the additive degree-Kirchhoff index. Yang and Klein [26] derived a formula for $R^{*}(G)$ of subdivisions and triangulations of graphs. To simplify the calculation of $R^{*}(G)$, the present authors [27] also obtained a formula for $R^{*}(G)$ with respect to the subgraph of $G$. For more work on the topological indices, we refer the reader to $[13,21,22,28-31]$.

In this paper, we study the multiplicative degree-Kirchhoff index of cacti. To state our results, we introduce some notation and terminology. For graph-theoretical terms that are not defined here, we refer to Bollobás' book [32]. Let $P_{n}, C_{n}$ and $S_{n}$ be the path, the cycle and the star on $n$ vertices, respectively. We denote by $G \cong H$ if graph $G$ is isomorphic to graph $H$. Let $N_{G}(x)=\{y \mid y x \in E\}$. Denote by $d_{G}(x)=\left|N_{G}(x)\right|$ the degree of the vertex $x$ of $G$. If $E_{0} \subset E$, we denote by $G-E_{0}$ the subgraph of $G$ obtained by deleting the edges in $E_{0}$. If $E_{1}$ is the subset of the edge set of the complement of $G, G+E_{1}$ denotes the graph obtained from $G$ by adding the edges in $E_{1}$. Similarly, if $W \subset V(G)$, we denote by $G-W$ the subgraph of $G$ obtained by deleting the vertices of $W$ and the edges incident with them and $G[W]$ the subgraph of $G$ induced by $W$. If $E=\{x y\}$ and $W=\{x\}$, we write $G-x y$ and $G-x$ instead of $G-\{x y\}$ and $G-\{x\}$, respectively.

A graph $G$ is called a cactus if each block of $G$ is either an edge or a cycle. Denote by $\operatorname{Cact}(n ; t)$ the set of cacti possessing $n$ vertices and $t$ cycles. Let $G \in \operatorname{Cact}(n ; t), t \geq 2$, a cycle $C=v_{1} v_{2} \cdots v_{k} v_{1}$ of $G$ is said to be an end cycle if all vertices $v_{1}, \cdots, v_{k-1}$ are of degree two, and the degree of vertex $v_{k}$ is greater than two. The vertex $v_{k} \in V(C)$ is called the anchor of $C$. Let $G^{0}(n ; t)$ be the graph shown in Figure 1 . In this paper, we first give some transformations on $R^{*}(G)$, and then, by these transformations, we determine the first-minimum and second-minimum multiplicative degree-Kirchhoff index in $\operatorname{Cact}(n ; t)$ and characterize the corresponding extremal graphs, respectively. 


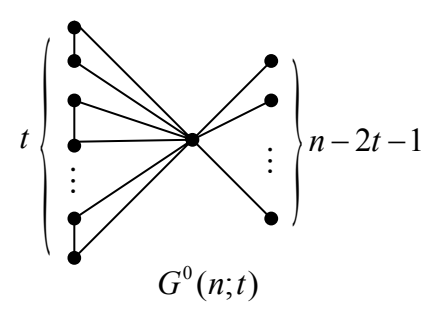

Figure 1. The graph $G^{0}(n ; t)$.

Now, we give some lemmas that are used in the proof of our main results.

Lemma 1. Ref. [13] Let $u$ be a cut vertex of a connected graph $G$ and $x$ and $y$ be vertices occurring in different components which arise upon deletion of $u$, then $r_{G}(x, y)=r_{G}(x, u)+r_{G}(u, y)$.

Lemma 2. Ref. [27] Let $G_{1}$ and $G_{2}$ be connected graphs with disjoint vertex sets, with $m_{1}$ and $m_{2}$ edges, respectively. Let $u_{1} \in V\left(G_{1}\right), u_{2} \in V\left(G_{2}\right)$. Constructing the graph $G$ by identifying the vertices $u_{1}$ and $u_{2}$, and denote the so obtained vertex by $u$. Then,

$$
R^{*}(G)=R^{*}\left(G_{1}\right)+R^{*}\left(G_{2}\right)+2 m_{2} \sum_{x \in V\left(G_{1}\right)} d_{G_{1}}(x) r_{G_{1}}(u, x)+2 m_{1} \sum_{y \in V\left(G_{2}\right)} d_{G_{2}}(y) r_{G_{2}}(u, y) .
$$

For completeness, we also give the proof in this paper.

Proof. Let $V_{1}=V\left(G_{1}\right)-u, V_{2}=V\left(G_{2}\right)-u$. Note that $\forall x \in V_{i}, d_{G}(x)=d_{G_{i}}(x)$ for $i=1,2$, and $d_{G}(u)=d_{G_{1}}(u)+d_{G_{2}}(u)$. By the definition of $R^{*}(G)$ and Lemma 1, we have

$$
\begin{aligned}
R^{*}(G)= & \frac{1}{2} \sum_{x, y \in V_{1}} d_{G}(x) d_{G}(y) r_{G}(x, y)+\frac{1}{2} \sum_{x, y \in V_{2}} d_{G}(x) d_{G}(y) r_{G}(x, y)+\sum_{x \in V_{1}} d_{G}(x) d_{G}(u) r_{G}(x, u)+ \\
& \sum_{y \in V_{2}} d_{G}(y) d_{G}(u) r_{G}(u, y)+\sum_{x \in V_{1}, y \in V_{2}} d_{G}(x) d_{G}(y) r_{G}(x, y) \\
= & {\left[R^{*}\left(G_{1}\right)-\sum_{x \in V_{1}} d_{G_{1}}(x) d_{G_{1}}(u) r_{G_{1}}(x, u)\right]+\left[R^{*}\left(G_{2}\right)-\sum_{y \in V_{2}} d_{G_{2}}(y) d_{G_{2}}(u) r_{G_{2}}(u, y)\right]+} \\
& \sum_{x \in V_{1}} d_{G_{1}}(x) d_{G}(u) r_{G}(x, u)+\sum_{y \in V_{2}} d_{G_{2}}(y) d_{G}(u) r_{G}(u, y)+ \\
& \sum_{x \in V_{1}, y \in V_{2}} d_{G_{1}}(x) d_{G_{2}}(y)\left[r_{G_{1}}(x, u)+r_{G_{2}}(u, y)\right]
\end{aligned}
$$


Because $d_{G}(u)=d_{G_{1}}(u)+d_{G_{2}}(u)$ and $r_{G}(x, u)=r_{G_{1}}(x, u)$ for $x \in V_{1}, r_{G}(u, y)=r_{G_{2}}(u, y)$ for $y \in V_{2}$, we have

$$
\begin{aligned}
R^{*}(G)= & {\left[R^{*}\left(G_{1}\right)+d_{G_{2}}(u) \sum_{x \in V_{1}} d_{G_{1}}(x) r_{G_{1}}(x, u)\right]+\left[R^{*}\left(G_{2}\right)+d_{G_{1}}(u) \sum_{y \in V_{2}} d_{G_{2}}(y) r_{G_{2}}(u, y)\right]+} \\
& \sum_{y \in V_{2}} \sum_{x \in V_{1}} d_{G_{1}}(x) d_{G_{2}}(y) r_{G_{1}}(x, u)+\sum_{x \in V_{1}} \sum_{y \in V_{2}} d_{G_{1}}(x) d_{G_{2}}(y) r_{G_{2}}(u, y) \\
= & {\left[R^{*}\left(G_{1}\right)+d_{G_{2}}(u) \sum_{x \in V_{1}} d_{G_{1}}(x) r_{G_{1}}(x, u)\right]+\left[R^{*}\left(G_{2}\right)+d_{G_{1}}(u) \sum_{y \in V_{2}} d_{G_{2}}(y) r_{G_{2}}(u, y)\right]+} \\
& \sum_{y \in V_{2}} d_{G_{2}}(y) \cdot \sum_{x \in V_{1}} d_{G_{1}}(x) r_{G_{1}}(x, u)+\sum_{x \in V_{1}} d_{G_{1}}(x) \cdot \sum_{y \in V_{2}} d_{G_{2}}(y) r_{G_{2}}(u, y) \\
= & {\left[R^{*}\left(G_{1}\right)+d_{G_{2}}(u) \sum_{x \in V_{1}} d_{G_{1}}(x) r_{G_{1}}(x, u)\right]+\left[R^{*}\left(G_{2}\right)+d_{G_{1}}(u) \sum_{y \in V_{2}} d_{G_{2}}(y) r_{G_{2}}(u, y)\right]+} \\
& {\left[2 m_{2}-d_{G_{2}}(u)\right] \sum_{x \in V_{1}} d_{G_{1}}(x) r_{G_{1}}(x, u)+\left[2 m_{1}-d_{G_{1}}(u)\right] \sum_{y \in V_{2}} d_{G_{2}}(y) r_{G_{2}}(u, y) } \\
= & R^{*}\left(G_{1}\right)+R^{*}\left(G_{2}\right)+2 m_{2} \sum_{x \in V\left(G_{1}\right)} d_{G_{1}}(x) r_{G_{1}}(u, x)+2 m_{1} \sum_{y \in V\left(G_{2}\right)} d_{G_{2}}(y) r_{G_{2}}(u, y),
\end{aligned}
$$

since $r_{G_{i}}(u, u)=0$ for $i=1,2$.

Lemma 3. Ref. [27] Let $G \in \mathscr{U}_{n}$, then $R^{*}(G) \geq R^{*}\left(G^{0}(n ; 1)\right)$, where $\mathscr{U}_{n}$ is the class of unicyclic graphs. The equality holds if and only if $T \cong G^{0}(n ; 1)$.

\section{Transformations}

In this section, we give some transformations that decrease $R^{*}(G)$.

Transformation 1. Let $u_{1} u_{2}$ be a cut-edge of $G$, but not an pendent edge, $G_{1}, G_{2}$ be the connected components of $G-u_{1} u_{2}$, where $u_{1} \in V\left(G_{1}\right), u_{2} \in V\left(G_{2}\right)$. Constructing the graph $G^{\prime}$ from $G$ by deleting $u_{1} u_{2}$ and identifying the vertices $u_{1}, u_{2}$, denote the so obtained vertex by $u$, adding an pendent edge uv (as shown in Figure 2).

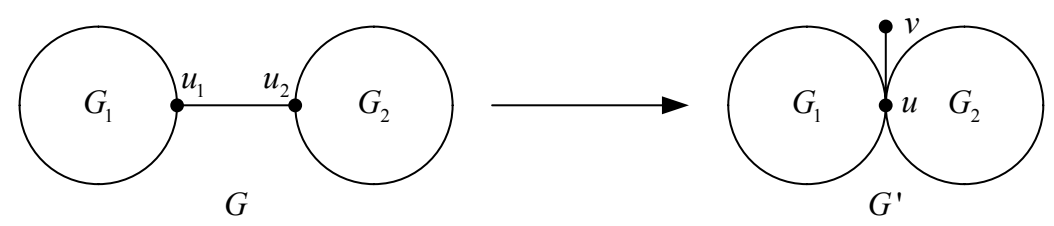

Figure 2. The graphs $G$ and $G^{\prime}$ in Transformation 1.

Lemma 4. Let $G, G^{\prime}$ be the graphs described in Transformation 1 , then $R^{*}(G)>R^{*}\left(G^{\prime}\right)$.

Proof. Let $\left|V\left(G_{1}\right)\right|=n_{1},\left|V\left(G_{2}\right)\right|=n_{2}$ and $\left|E\left(G_{1}\right)\right|=m_{1},\left|E\left(G_{2}\right)\right|=m_{2}$, where $m_{1}, m_{2} \geq 1$. Let $H$ be the graph obtained by attaching to the vertex $u_{1}$ of $G_{1}$ the pendent vertex $u_{2}$, then $|V(H)|=$ $n_{1}+1,|E(H)|=m_{1}+1$. By Lemma 2, we have

$$
\begin{aligned}
R^{*}(G) & =R^{*}(H)+R^{*}\left(G_{2}\right)+2 m_{2} \sum_{x \in V(H)} d_{H}(x) r_{H}\left(u_{2}, x\right)+2\left(m_{1}+1\right) \sum_{y \in V\left(G_{2}\right)} d_{G_{2}}(y) r_{G_{2}}\left(u_{2}, y\right), \\
R^{*}\left(G^{\prime}\right) & =R^{*}(H)+R^{*}\left(G_{2}\right)+2 m_{2} \sum_{x \in V(H)} d_{H}(x) r_{H}(u, x)+2\left(m_{1}+1\right) \sum_{y \in V\left(G_{2}\right)} d_{G_{2}}(y) r_{G_{2}}(u, y) .
\end{aligned}
$$


Note that $r_{G_{2}}\left(u_{2}, y\right)=r_{G_{2}}(u, y)$, then

$$
\begin{aligned}
R^{*}(G)-R^{*}\left(G^{\prime}\right) & =2 m_{2}\left[\sum_{x \in V(H)} d_{H}(x) r_{H}\left(u_{2}, x\right)-\sum_{x \in V(H)} d_{H}(x) r_{H}(u, x)\right] \\
& =2 m_{2}\left\{\sum_{x \in V\left(G_{1}\right)} d_{H}(x)\left[r_{H}\left(u_{1}, x\right)+1\right]-\left[\sum_{x \in V\left(G_{1}\right)} d_{H}(x) r_{H}(u, x)+1\right]\right\} \\
& =2 m_{2}\left[2\left(m_{1}+1\right)-1-1\right]=4 m_{1} m_{2}>0,
\end{aligned}
$$

so $R^{*}(G)>R^{*}\left(G^{\prime}\right)$.

Let $\mathscr{G}_{n}$ be the class of connected graphs on $n$ vertices. By Transformation 1 and Lemma 4, we have the following result.

Corollary 1. Let $G_{0}$ be a graph with the smallest multiplicative degree-Kirchhoff index in $\mathscr{G}_{n}$, then all cut-edges are pendent edges.

Transformation 2. For $G \in \operatorname{Cact}(n ; t)$, let $C_{k}$ be a cycle with $k(\geq 4)$ vertices, contained in $G$. Let there be a unique vertex $u \in C_{h}$ which is adjacent to a vertex in $V(G)-V(C)$. Assuming that $u v, v w \in E(C)$, construct a new graph $G^{*}=G-v w+u w$ (as shown in Figure 3 ).

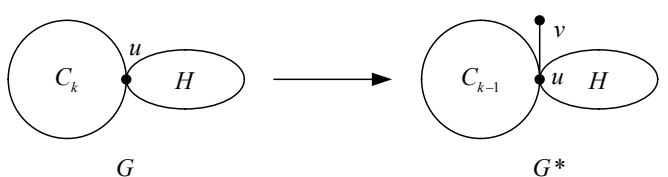

Figure 3. The graphs $G$ and $G^{*}$ in Transformation 2.

For $u \in V\left(C_{k}\right)$, by direct calculation, we have

$$
\begin{aligned}
R^{*}\left(C_{k}\right) & =\frac{k^{3}-k}{3} \\
\sum_{y \in V\left(C_{k}\right)} r_{C_{k}}(u, y) & =\frac{k^{2}-1}{6}
\end{aligned}
$$

Lemma 5. Let $G, G^{*}$ be the graphs described in Transformation 2 , then $R^{*}(G)>R^{*}\left(G^{*}\right)$.

Proof. Let $S$ be the graph obtained by attaching to the vertex $u$ of $C_{k-1}$ the pendent vertex $v$. By Lemma 1, we have

$$
\begin{aligned}
R^{*}(G) & =R^{*}(H)+R^{*}\left(C_{k}\right)+2 k \sum_{x \in V(H)} d_{H}(x) r_{H}(u, x)+2|E(H)| \sum_{y \in V\left(C_{k}\right)} d_{C_{k}}(y) r_{C_{k}}(u, y), \\
R^{*}\left(G^{*}\right) & =R^{*}(H)+R^{*}(S)+2 k \sum_{x \in V(H)} d_{H}(x) r_{H}(u, x)+2|E(H)| \sum_{y \in V(S)} d_{S}(y) r_{S}(u, y) .
\end{aligned}
$$

Further by Equations (1) and (2), then

$$
\begin{aligned}
R^{*}(G)-R^{*}\left(G^{*}\right) & =\left[R^{*}\left(C_{k}\right)-R^{*}(S)\right]+2|E(H)|\left[\sum_{y \in V\left(C_{k}\right)} d_{C_{k}}(y) r_{C_{k}}(u, y)-\sum_{y \in V(S)} d_{S}(y) r_{S}(u, y)\right] \\
& =\left[\frac{k^{3}-k}{3}-\left(\frac{(k-1)\left(k^{2}+2\right)}{3}+2 k-1\right)\right]+2|E(H)| \cdot \frac{2 k-4}{3} \\
& \geq \frac{k^{2}-5 k+3}{3}+\frac{4 k-8}{3}=\frac{k^{2}-k-5}{3}>0
\end{aligned}
$$

since $k \geq 4,|E(H)| \geq 1$. 
Transformation 3. Let $G \in \operatorname{Cact}(n ; t), t \geq 2$, be a cactus without cut edges. Let $C$ be an end cycle of $G$ and $u$ be its anchor. Let $v$ be a vertex of $C$ different from $u$. The graphs $G_{1}$ and $G_{2}$ are constructed by adding $r$ pendent edges to the vertices $u$ and $v$ of $G$ respectively (as shown in Figure 4).

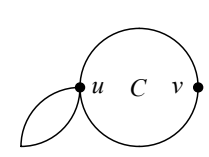

G

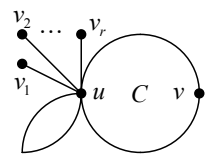

$G_{1}$

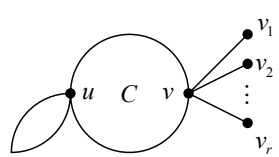

$G_{2}$

Figure 4. The graphs $G, G_{1}$ and $G_{3}$ in Transformation 3 .

Lemma 6. Let $G, G_{1}, G_{2}$ be the graphs described in Transformation 3, then $R^{*}\left(G_{2}\right)>R^{*}\left(G_{1}\right)$.

Proof. Let $H=G[V(G)-V(C)], H_{1}=G\left[V\left(G_{1}\right) \backslash(V(G)-u)\right]$ and $H_{2}=G\left[V\left(G_{2}\right) \backslash(V(G)-v)\right]$, then $H_{1} \cong H_{2} \cong K_{1, r}$. By Lemma 2 , we have

$$
\begin{aligned}
& R^{*}\left(G_{1}\right)=R^{*}(G)+R^{*}\left(K_{1, r}\right)+2 r \sum_{x \in V(G)} d_{G}(x) r_{G}(u, x)+2|E(G)| \sum_{y \in V\left(K_{1, r}\right)} d_{K_{1, r}}(y) r_{K_{1, r}}(u, y), \\
& R^{*}\left(G_{2}\right)=R^{*}(G)+R^{*}\left(K_{1, r}\right)+2 r \sum_{x \in V(G)} d_{G}(x) r_{G}(v, x)+2|E(G)| \sum_{y \in V\left(K_{1, r}\right)} d_{K_{1, r}}(y) r_{K_{1, r}}(v, y) .
\end{aligned}
$$

Note that $r_{G}(u, u)=r_{G}(v, v)=0, V(G)=V(H) \cup\{V(C)-u\} \cup\{u\}$ and $r_{G}(v, x)=r_{G}(x, u)+$ $r_{G}(v, u)$ for $x \in V(H)$, then

$$
\begin{aligned}
R^{*}\left(G_{2}\right)-R^{*}\left(G_{1}\right)= & 2 r\left[\sum_{x \in V(G)} d_{G}(x) r_{G}(v, x)-\sum_{x \in V(G)} d_{G}(x) r_{G}(u, x)\right] \\
= & 2 r\left\{\left[\sum_{x \in V(H)} d_{G}(x)\left(r_{G}(x, u)+r_{G}(v, u)\right)+\sum_{x \in V(C)-u} d_{G}(x) r_{G}(v, x)+d_{G}(u) r_{G}(v, u)\right]\right. \\
& \left.-\left[\sum_{x \in V(H)} d_{G}(x) r_{G}(u, x)+\sum_{x \in V(C)-u} d_{G}(x) r_{G}(u, x)\right]\right\}
\end{aligned}
$$

Considering that $d_{G}(u)=d_{H}(u)+d_{C}(u)$ and $\sum_{x \in V(C)-u} d_{G}(x) r_{G}(u, x)=$ $\left.\sum_{x \in V(C)-u} d_{G}(x) r_{G}(v, x)+d_{C}(u) r_{G}(v, u)\right]$, we obtain

$$
R^{*}\left(G_{2}\right)-R^{*}\left(G_{1}\right)=2 r\left[\sum_{x \in V(H)} d_{G}(x) r_{G}(v, u)+d_{G}(u) r_{G}(v, u)\right]>0 .
$$

This completes the proof.

Transformation 4. Let $C=v_{1} v_{2} v_{3} v_{1}$ be a cycle of the graph $G, C$ is called a pendant triangle if $d_{G}\left(v_{1}\right)=$ $d_{G}\left(v_{2}\right)=2$ and $d_{G}\left(v_{3}\right)>2$. Suppose that $G$ is a cactus graph and $u, v \in V(G)$ are two vertices, such that $u x_{i} y_{i} u(i=1,2, \ldots, s)$ and $v p_{j} q_{j} v(j=1,2, \ldots, t)$ are pendant triangles with the anchor $u$ and $v$, respectively. We form two new graphs $A$ and $B$ according to the following transformation.

$$
\begin{aligned}
A & =G-\cup_{i=1}^{s}\left\{u x_{i}, u y_{i}\right\}+\cup_{i=1}^{s}\left\{v x_{i}, v y_{i}\right\}, \\
B & =G-\cup_{i=1}^{t}\left\{v p_{i}, v q_{i}\right\}+\cup_{i=1}^{t}\left\{u p_{i}, u q_{i}\right\} .
\end{aligned}
$$

Lemma 7. Let $G, A, B$ be the graphs described in Transformation 4, then either $R^{*}(G)>R^{*}(A)$ or $R^{*}(G)>$ $R^{*}(B)$. 
Proof. Let $M=\left\{x_{1}, y_{1}, \ldots, x_{s}, y_{s}\right\}, N=\left\{p_{1}, q_{1}, \ldots, p_{t}, q_{t}\right\}$ and $H=V(G)-M-N-\{u, v\}$. Because of $V(G)=M \cup N \cup H \cup\{u, v\}$, by Lemma 2, we have

$$
\begin{aligned}
R^{*}(G)= & {\left[\sum_{\{x, y\} \subset M}+\sum_{\{x, y\} \subset N}+\sum_{\{x, y\} \subset H}\right] d_{G}(x) d_{G}(y) r_{G}(x, y)+\sum_{x \in H, y \in M} d_{G}(x) d_{G}(y) r_{G}(x, y)+} \\
& \sum_{x \in H, y \in N} d_{G}(x) d_{G}(y) r_{G}(x, y)+\sum_{x \in M, y \in N} d_{G}(x) d_{G}(y) r_{G}(x, y)+d_{G}(u) d_{G}(v) r_{G}(u, v) \\
& +\sum_{x \in H} d_{G}(x) d_{G}(u) r_{G}(x, u)+\sum_{x \in H} d_{G}(x) d_{G}(v) r_{G}(x, v)+\sum_{x \in M} d_{G}(x) d_{G}(u) r_{G}(x, u)+ \\
& \sum_{x \in M} d_{G}(x) d_{G}(v) r_{G}(x, v)+\sum_{x \in N} d_{G}(x) d_{G}(u) r_{G}(x, u)+\sum_{x \in N} d_{G}(x) d_{G}(v) r_{G}(x, v) .
\end{aligned}
$$

and analogously

$$
\begin{aligned}
R^{*}(A)= & {\left[\sum_{\{x, y\} \subset M}+\sum_{\{x, y\} \subset N}+\sum_{\{x, y\} \subset H}\right] d_{A}(x) d_{A}(y) r_{A}(x, y)+\sum_{x \in H, y \in M} d_{A}(x) d_{A}(y) r_{A}(x, y)+} \\
& \sum_{x \in H, y \in N} d_{A}(x) d_{A}(y) r_{A}(x, y)+\sum_{x \in M, y \in N} d_{A}(x) d_{A}(y) r_{A}(x, y)+d_{A}(u) d_{A}(v) r_{A}(u, v) \\
& +\sum_{x \in H} d_{A}(x) d_{A}(u) r_{A}(x, u)+\sum_{x \in H} d_{A}(x) d_{A}(v) r_{A}(x, v)+\sum_{x \in M} d_{A}(x) d_{A}(u) r_{A}(x, u)+ \\
& \sum_{x \in M} d_{G}(x) d_{A}(v) r_{A}(x, v)+\sum_{x \in N} d_{A}(x) d_{A}(u) r_{A}(x, u)+\sum_{x \in N} d_{A}(x) d_{A}(v) r_{A}(x, v) .
\end{aligned}
$$

Note that $d_{G}(x)=d_{A}(x)$ for $x \in V(G)-\{u, v\}$ and $r_{G}(x, y)=r_{A}(x, y)$ for $x, y \in M$ or $x, y \in N$ or $x, y \in H$, then

$$
\begin{aligned}
a_{0} & =\left[\sum_{\{x, y\} \subset M}+\sum_{\{x, y\} \subset N}+\sum_{\{x, y\} \subset H}\right] d_{G}(x) d_{G}(y) r_{G}(x, y), \\
b_{0} & =\left[\sum_{\{x, y\} \subset M}+\sum_{\{x, y\} \subset N}+\sum_{\{x, y\} \subset H}\right] d_{A}(x) d_{A}(y) r_{A}(x, y), \\
a_{0}-b_{0} & =0
\end{aligned}
$$

Considering that $r(u, y)=\frac{2}{3}$ for $y \in M$ and $r(x, y)=r(x, u)+r(u, y)$, we get

$$
\begin{aligned}
a_{1}=\sum_{x \in H, y \in M} d_{G}(x) d_{G}(y) r_{G}(x, y) & =\sum_{x \in H, y \in M} d_{G}(x) d_{G}(y)\left[r_{G}(x, u)+\frac{2}{3}\right], \\
b_{1}=\sum_{x \in H, y \in M} d_{A}(x) d_{A}(y) r_{A}(x, y) & =\sum_{x \in H, y \in M} d_{G}(x) d_{G}(y)\left[r_{G}(x, v)+\frac{2}{3}\right], \\
a_{1}-b_{1} & =\sum_{x \in H, y \in M} d_{G}(x) d_{G}(y)\left[r_{G}(x, u)-r_{G}(x, v)\right]
\end{aligned}
$$

and analogously,

$$
\begin{aligned}
a_{2}=\sum_{x \in H, y \in N} d_{G}(x) d_{G}(y) r_{G}(x, y) & =\sum_{x \in H, y \in N} d_{G}(x) d_{G}(y)\left[r_{G}(x, v)+\frac{2}{3}\right], \\
b_{2}=\sum_{x \in H, y \in N} d_{A}(x) d_{A}(y) r_{A}(x, y) & =\sum_{x \in H, y \in N} d_{G}(x) d_{G}(y)\left[r_{G}(x, v)+\frac{2}{3}\right], \\
a_{2}-b_{2} & =0
\end{aligned}
$$

Because $r_{G}(x, y)=r_{G}(x, u)+r_{G}(u, v)+r_{G}(v, y)=r_{G}(u, v)+\frac{4}{3}$ for $x \in M, y \in N$, then

$$
\begin{aligned}
a_{3}=\sum_{x \in M, y \in N} d_{G}(x) d_{G}(y) r_{G}(x, y) & =\sum_{x \in M, y \in N} d_{G}(x) d_{G}(y)\left[r_{G}(u, v)+\frac{4}{3}\right], \\
b_{3}=\sum_{x \in M, y \in N} d_{A}(x) d_{A}(y) r_{A}(x, y) & =\frac{4}{3} \sum_{x \in M, y \in N} d_{G}(x) d_{G}(y), \\
a_{3}-b_{3} & =\sum_{x \in M, y \in N} d_{G}(x) d_{G}(y) r_{G}(u, v)
\end{aligned}
$$




$$
\begin{aligned}
& a_{4}= d_{G}(u) d_{G}(v) r_{G}(u, v), \\
& b_{4}=d_{A}(u) d_{A}(v) r_{A}(u, v)= {\left[d_{G}(u)-2 s\right]\left[d_{G}(v)+2 s\right] r_{G}(u, v), } \\
& a_{4}-b_{4}=4 s^{2} r_{G}(u, v)+2 s d_{G}(v) r_{G}(u, v)-2 s d_{G}(u) r_{G}(u, v) a_{5}=\sum_{x \in H} d_{G}(x) d_{G}(u) r_{G}(x, u), \\
& b_{5}=\sum_{x \in H} d_{A}(x) d_{A}(u) r_{A}(x, u)=\sum_{x \in H} d_{G}(x)\left[d_{G}(u)-2 s\right] r_{G}(x, u), \\
& a_{5}-b_{5}=\sum_{x \in H} 2 s d_{G}(x) r_{G}(x, u) \\
& a_{6}=\sum_{x \in H} d_{G}(x) d_{G}(v) r_{G}(x, v), \\
& a_{6}-b_{6}=-\sum_{x \in H} 2 s d_{G}(x) r_{G}(x, v) \\
& b_{7 \in H} d_{A}(x) d_{A}(v) r_{A}(x, v) \sum_{x \in H} d_{G}(x)\left[d_{G}(v)+2 s\right] r_{G}(x, v), \\
& a_{7}=\sum_{x \in M} d_{G}(x) d_{G}(u) r_{G}(x, u)= \frac{2}{3} \sum_{x \in M} d_{G}(x) d_{G}(u), \\
& b_{x \in M}(x) d_{A}(u) r_{A}(x, u)=\sum_{x \in M} d_{G}(x)\left[d_{G}(u)-2 s\right]\left[r_{G}(u, v)+\frac{2}{3}\right], \\
& a_{7}-b_{7}=\sum_{x \in M}\left[\left(2 s-d_{G}(u)\right) d_{G}(x) r_{G}(u, v)+\frac{4}{3} s d_{G}(x)\right]
\end{aligned}
$$

Because $r_{G}(x, v)=r_{G}(u, v)+\frac{2}{3}$ for $x \in M$. After the transformation, the degree of the vertex $v$ increases by $2 s$, and $r_{A}(x, v)=\frac{2}{3}$ for $x \in M$.

$$
\begin{aligned}
& a_{8}=\sum_{x \in M} d_{G}(x) d_{G}(v) r_{G}(x, v)=\sum_{x \in M} d_{G}(x) d_{G}(v)\left[r_{G}(u, v)+\frac{2}{3}\right], \\
& b_{8}=\sum_{x \in M} d_{G}(x) d_{A}(v) r_{A}(x, v)=\frac{2}{3} \sum_{x \in M} d_{G}(x)\left[d_{G}(v)+2 s\right], \\
& a_{8}-b_{8}=\sum_{x \in M}\left[d_{G}(x) d_{G}(v) r_{G}(u, v)-\frac{4}{3} s d_{G}(x)\right] \\
& a_{9}=\sum_{x \in N} d_{G}(x) d_{G}(u) r_{G}(x, u)=\sum_{x \in N} d_{G}(x) d_{G}(u)\left[r_{G}(v, u)+\frac{2}{3}\right], \\
& b_{9}=\sum_{x \in N} d_{A}(x) d_{A}(u) r_{A}(x, u)=\sum_{x \in N} d_{G}(x)\left[d_{G}(u)-2 s\right]\left[r_{G}(v, u)+\frac{2}{3}\right], \\
& a_{9}-b_{9}=\sum_{x \in N} 2 s d_{G}(x)\left[r_{G}(v, u)+\frac{2}{3}\right] \\
& a_{10}=\sum_{x \in N} d_{G}(x) d_{G}(v) r_{G}(x, v)=\frac{2}{3} \sum_{x \in N} d_{G}(x) d_{G}(v), \\
& b_{10}=\sum_{x \in N} d_{A}(x) d_{A}(v) r_{A}(x, v)=\frac{2}{3} \sum_{x \in N} d_{G}(x)\left[d_{G}(v)+2 s\right], \\
& a_{10}-b_{10}=-\frac{4}{3} \sum_{x \in N} s d_{G}(x)
\end{aligned}
$$

By Equations (3)-(13), we have

$$
\begin{aligned}
R^{*}(G)-R^{*}(A)= & 4 s \sum_{x \in H} d_{G}(x)\left[r_{G}(x, u)-r_{G}(x, v)\right]+24 s t r_{G}(u, v)+6 s r_{G}(u, v)\left[d_{G}(v)-d_{G}(u)\right]+ \\
& 12 s^{2} r_{G}(u, v)+\sum_{x \in H} 2 s d_{G}(x)\left[r_{G}(x, u)-r_{G}(x, v)\right] .
\end{aligned}
$$


Similarly, we have

$$
\begin{aligned}
R^{*}(G)-R^{*}(B)= & 4 t \sum_{x \in H} d_{G}(x)\left[r_{G}(x, v)-r_{G}(x, u)\right]+24 s \operatorname{tr}_{G}(u, v)+6 \operatorname{tr}_{G}(u, v)\left[d_{G}(u)-d_{G}(v)\right]+ \\
& 12 t^{2} r_{G}(u, v)+2 t \sum_{x \in H} d_{G}(x)\left[r_{G}(x, v)-r_{G}(x, u)\right] .
\end{aligned}
$$

If $R^{*}(G)-R^{*}(A) \leq 0$, then

$$
\begin{aligned}
& 4 \sum_{x \in H} d_{G}(x)\left[r_{G}(x, u)-r_{G}(x, v)\right]+6 r_{G}(u, v)\left[d_{G}(v)-d_{G}(u)\right]+\sum_{x \in H} 2 d_{G}(x)\left[r_{G}(x, u)-r_{G}(x, v)\right] \\
\leq & -\left(24 \operatorname{tr}_{G}(u, v)+12 s r_{G}(u, v)\right) .
\end{aligned}
$$

Further, we have

$$
R^{*}(G)-R^{*}(B) \geq t\left(24 \operatorname{tr}_{G}(u, v)+12 s r_{G}(u, v)\right)+24 s \operatorname{st}_{G}(u, v)+12 t^{2} r_{G}(u, v)>0 .
$$

This completes the proof.

Transformation 5. Let $u$ be a vertex of $G$ such that there are s pendent vertices $u_{1}, u_{2}, \ldots, u_{s}$ attached to $u$, and $v$ be another vertex of $G$ such that there are $t$ pendent vertices $v_{1}, v_{2}, \ldots, v_{t}$ attached to $v$. Let

$$
\begin{aligned}
& G_{3}=G-\left\{u u_{1}, u u_{2}, \ldots, u u_{s}\right\}+\left\{v u_{1}, v u_{2}, \ldots, v u_{s}\right\} \\
& G_{4}=G-\left\{v v_{1}, v v_{2}, \ldots, v v_{t}\right\}+\left\{u v_{1}, u v_{2}, \ldots, u v_{t}\right\} .
\end{aligned}
$$

Similar to the proof of Lemma 7, we can prove the following result.

Lemma 8. Let $G, G_{3}$ and $G_{4}$ be graphs as described in Transformation 5, then either $R^{*}(G)>R^{*}\left(G_{3}\right)$ or $R^{*}(G)>R^{*}\left(G_{4}\right)$.

\section{Main Results}

In this section, we determine the elements in $\operatorname{Cact}(n ; t)$ with first-minimum and second-minimum multiplicative degree-Kirchhoff index by the transformations that we have obtained. Note that the first-minimum multiplicative degree-Kirchhoff index has been obtained in [33]; for completeness, we also give the following proof.

Theorem 1. Ref. [33] Let $G \in \operatorname{Cact}(n ; t)$, then $R^{*}(G) \geq 16 t^{2}-8 t+(n-2 t-1)^{2}+(n-2 t-1)(n-$ $2 t-2)+\frac{34}{3} t(n-2 t-1)$. The equality holds if and only if $G \cong G^{0}(n, t)$.

Proof. Let $\tilde{G}$ be the unique graph having the minimum multiplicative degree-Kirchhoff index in $\operatorname{Cact}(n ; t)$.

Case 1. If $t=1, \operatorname{Cact}(n ; t)$ is the class of unicyclic graphs. By Lemma 3, we know the results hold.

Case 2. If $t=2, \operatorname{Cact}(n ; t)$ is the class of bicyclic graphs. By Lemma 4 , we conclude that $\tilde{G}$ contains two cycles attached to a common vertex $u$, and all cut-edges are all pendent edges (if any). Further, by Lemmas 8 and 6, all pendent edges (if any) are also attached to $u$. Finally, by Lemma 5, the two cycles must be triangles, that is, $\tilde{G} \cong G^{0}(n, 2)$. This obtains the desirable results.

Case 3. If $t \geq 3$, by Lemma 4, we conclude that all cut-edges are all pendent edges (if any) in $\tilde{G}$. Further, by Lemmas 8 and $6, \tilde{G}$ has at least two end cycles. Repeated by Lemmas $5-8$, we arrive at the conclusion $\tilde{G} \cong G^{0}(n, t)$

By direct calculation, we have

$$
R^{*}\left(G^{0}(n ; t)\right)=16 t^{2}-8 t+(n-2 t-1)^{2}+(n-2 t-1)(n-2 t-2)+\frac{34}{3} t(n-2 t-1) .
$$


This completes the proof.

Theorem 2. Let $G \in \operatorname{Cact}(n ; t) \backslash G^{0}(n ; t)$, then $R^{*}(G) \geq\left[16 t^{2}+\frac{34 t-22}{3}\right]+\left[(n-2 t-2)^{2}+(n-2 t-\right.$ $2)(n-2 t-3)]+\frac{(16 t+14)(n-2 t-2)}{3}+2(3 t+1)(n-2 t-2)$. The equality holds if and only if $G \cong G_{3}^{0}$.

Proof. By Lemmas 4-8 and Theorem 1, one can conclude that $G$, which has the second-minimum multiplicative degree-Kirchhoff index in $\operatorname{Cact}(n ; t)$ must be one of the graphs $G_{1}^{0}, G_{2}^{0}, G_{3}^{0}$, as shown in Figure 5. By Lemma 2, we have

$$
\begin{aligned}
R^{*}\left(G_{1}^{0}\right)= & {\left[16 t^{2}-8 t\right]+[2+4(n-2 t-2)+7(n-2 t-3)+(n-2 t-2)(n-2 t-3)+} \\
& (n-2 t-3)(n-2 t-4)]+\frac{16}{3} t(n-2 t-1)+6 t(n-2 t+1), \\
R^{*}\left(G_{2}^{0}\right)= & {\left[16 t^{2}+\frac{34}{3} t-7\right]+\left[(n-2 t-2)^{2}+(n-2 t-2)(n-2 t-3)\right]+\frac{(16 t+14)(n-2 t-2)}{3} } \\
& +2(3 t+1)(n-2 t-2), \\
R^{*}\left(G_{3}^{0}\right)= & {\left[16 t^{2}+\frac{34 t-22}{3}\right]+\left[(n-2 t-2)^{2}+(n-2 t-2)(n-2 t-3)\right]+\frac{(16 t+14)(n-2 t-2)}{3} } \\
& +2(3 t+1)(n-2 t-2) .
\end{aligned}
$$

Then,

$$
\begin{aligned}
& R^{*}\left(G_{1}^{0}\right)-R^{*}\left(G_{3}^{0}\right)=\frac{4 n+4 t+5}{3}, \\
& R^{*}\left(G_{2}^{0}\right)-R^{*}\left(G_{3}^{0}\right)=\frac{1}{3} .
\end{aligned}
$$

This completes the proof.

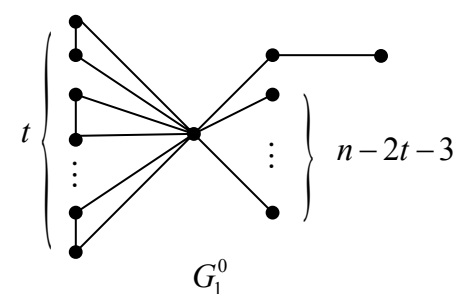

$G_{1}^{0}$

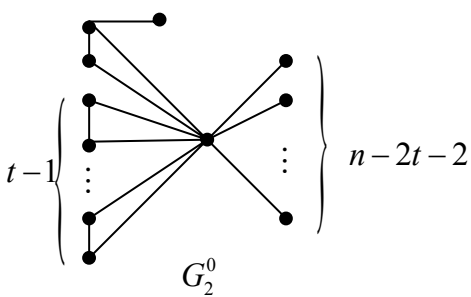

Figure 5. The graphs $G_{1}^{0}, G_{2}^{0}, G_{3}^{0}$.

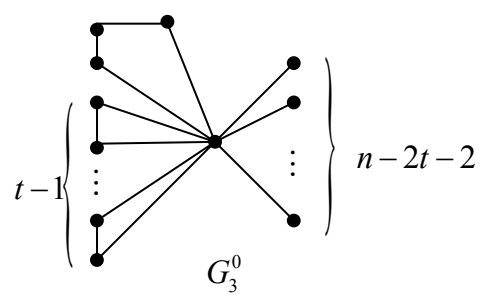

$G_{3}^{0}$

By Theorems 1 and 2, we have

Corollary 2. Among all graphs in $\operatorname{Cact}(n ; t), G^{0}(n ; t)$ and $G_{3}^{0}$ are the graphs with first-minimum and second-minimum multiplicative degree-Kirchhoff index.

According to the above discussion, we find that the extremal cacti for the index $R^{*}(G)$ are the same as the extremal cacti for the Kirchhoff index, the multiplicative degree-Kirchhoff index, the Wiener index and the other indices [22,29,34,35]. Based on the known results for these indices, we guess the element of $\operatorname{Cact}(n ; t)$ with maximum multiplicative degree-Kirchhoff index is isomorphic to the graph $C_{n, t}$ (as shown in Figure 6). 


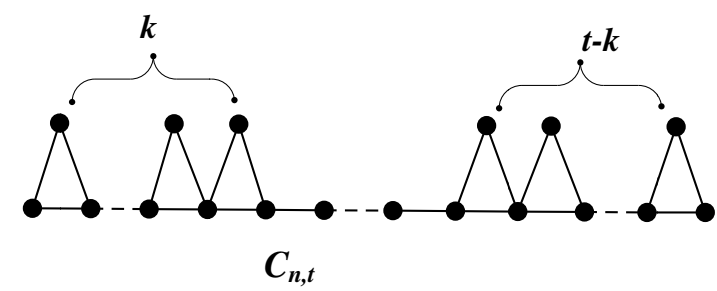

Figure 6. The graphs $C_{n, t}$.

Conjecture 1. Let $C_{n, t}$ be the graph depicted in Figure 6, where $k=\left\lfloor\frac{t}{2}\right\rfloor$. Then, $C_{n, t}$ is the unique element of Cact $(n ; t)$ having maximum multiplicative degree-Kirchhoff index.

In particular, for $\operatorname{Cat}(n ; t)$, if $t=1 ; 0, \operatorname{Cat}(n ; 1)$ and $\operatorname{Cat}(n ; 0)$ are the set of unicyclic graphs and trees, respectively. For $G \in \operatorname{Cat}(n ; 1)$, the graphs having maximum and minimum multiplicative degree-Kirchhoff index are given in [13], that is

$$
R^{*}\left(G^{0}(n, 1)\right) \leq R^{*}(G) \leq R^{*}\left(U_{3}^{n}\right) .
$$

where $U_{3}^{n}$ consists of a cycle of size 3 to which a path with $n-3$ vertices is attached. For $G \in \operatorname{Cat}(n ; 0)$, it is easy to get the result

$$
R^{*}\left(S_{n}\right) \leq R^{*}(G) \leq R^{*}\left(P_{n}\right) .
$$

\section{Conclusions}

In this paper, we give some transformations on the multiplicative degree-Kirchhoff index. As applications, the second-minimum multiplicative degree-Kirchhoff index on Cat $(n ; t)$ and the corresponding extremal graph are determined. We guess $C_{n, t}$ is the graph of $C a t(n ; t)$ with maximum $R^{*}(G)$ value. For solving the problem, our approach would need to be modified; it would be interesting to continue studying the extremal graphs.

Author Contributions: Investigation, Z.Z.; Methodology, F.H.

Funding: This research received no external funding.

Conflicts of Interest: The authors declare no conflict of interest.

\section{References}

1. Wiener, H. Structural determination of paraffin boiling points. J. Am. Chem. Soc. 1947, 69, 17-20. [CrossRef] [PubMed]

2. Dobrynin, A.A.; Kochetova, A.A. Degree distance of a graph: A degree analogue of the Wiener index. J. Chem. Inf. Comput. Sci. 1994, 34, 1082-1086. [CrossRef]

3. Ali, P.; Mukwembi, S.; Munyira, S. Degree distance and vertex-connectivity. Discret. Appl. Math. 2013, 161, 2802-2811. [CrossRef]

4. Dankelmannn, P.; Gutman, I.; Mukwembi, S.; Swart, H.C. On the degree distance of a graph. Discret. Appl. Math. 2009, 157, 2773-2777. [CrossRef]

5. Li, S.C.; Song, Y.B.; Zhang, H.H. On the degree distance of unicyclic graphs with given matching number. Graphs Comb. 2015, 31, 2261-2274. [CrossRef]

6. Tomescu, I. Ordering connected graphs having small degree distances. Discret. Appl. Math. 2010, 158, 1714-1717. [CrossRef]

7. Schultz, H.P. Topological organic chemistry. 1. Graph theory and topological indices of alkanes. J. Chem. Inf. Comput. Sci. 1989, 29, 227-228. [CrossRef]

8. Gutman, I.; Das, K.C. The first Zagreb index 30 years after. MATCH Commun. Math. Comput. Chem. 2004, 50, 83-92.

9. Klein, D.J.; Mihalić, Z.; Plavšić, D.; Trinajstić, N. Molecular topological index: A relation with the Wiener index. J. Chem. Inf. Comput. Sci. 1992, 32, 304-305. [CrossRef] 
10. Gutman, I. Selected properties of the Schultz molecular topological index. J. Chem. Inf. Comput. Sci. 1994, 34, 1087-1089. [CrossRef]

11. Agnes, V.S. Degree distance and Gutman index of corona product of graphs. Trans. Comb. 2015, 4, 11-23.

12. Knor, M.; Potočnik, P.; Škrekovski, R. Relationship between the edge Wiener index and the Gutman index of a graph. Discret. Appl. Math. 2014, 167, 197-201. [CrossRef]

13. Klein, D.J.; Randić, M. Resistance distance. J. Math. Chem. 1993, 12, 81-95. [CrossRef]

14. Arauz, C. The Kirchhoff indexes of some composite networks. Discret. Appl. Math. 2012, 160, 1429-1440. [CrossRef]

15. Feng, L.; Yu, G.; Xu, K.; Jiang, Z. A note on the Kirchhoff index of bicyclic graphs. Ars Comb. 2014, 114, 33-40.

16. Yang, Y.; Jiang, X. Unicyclic graphs with extremal Kirchhoff index. MATCH Commun. Math. Comput. Chem. 2008, 60, 107-120.

17. Zhang, H.; Jiang, X.; Yang, Y. Bicyclic graphs with extremal Kirchhoff index. MATCH Commun. Math. Comput. Chem. 2009, 61, 697-712.

18. Zhou, B.; Trinajstić, N. On resistance-distance and kirchhoff index. J. Math. Chem. 2009, 46, $283-289$. [CrossRef]

19. Gutman, I.; Mohar, B. The quasi-Wiener and the Kirchhoff indices coincide. J. Chem. Inf. Comput. Sci. 1996, 36, 982-985. [CrossRef]

20. Gutman, I.; Feng, L.; Yu, G. Degree resistance distance of unicyclic graphs. Trans. Comb. 2012, 1, $27-40$.

21. Chen, H.; Zhang, F. Resistance distance and the normalized Laplacian spectrum. Discret. Appl. Math. 2007, 155, 654-661. [CrossRef]

22. Du, J.; Su, G.; Tu, J.; Gutman, I. The degree resistance distance of cacti. Discret. Appl. Math. 2015, 188, 16-24. [CrossRef]

23. Bianchi, M.; Cornaro, A.; Palacios, J.L.; Torriero, A. New upper and lower bounds for the additive degree-Kirchhoff index. Croat. Chem. Acta 2013, 86, 363-370. [CrossRef]

24. Feng, L.H.; Gutman, I.; Yu, G.H. Degree Kirchhoff index of unicyclic graphs. MATCH Commun. Math. Comput. Chem. 2013, 69, 629-648.

25. Palacios, J.L. Some interplay of the three Kirchhoffian indices. MATCH Commun. Math. Comput. Chem. 2016, 75, 199-206. [CrossRef]

26. Yang, Y.; Klein, D.J. Resistance distance-based graph invariants of subdivisions and triangulations of graphs. Discret. Appl. Math. 2015, 181, 260-274. [CrossRef]

27. Zhu, Z.; Hong, Y. Some transformations on multiplicative degree-Kirchhoff index of graphs and their applications. 2019, submitted.

28. Bianchi, M.; Cornaro, A.; Palacios, J.L.; Renom, J.M.; Torriero, A. Revisiting Bounds for the Multiplicative Degree-Kirchhoff Index. MATCH Commun. Math. Comput. Chem. 2016, 75, 227-231.

29. Liu, J.; Wanga, W.; Zhang, Y.; Pan, X. On degree resistance distance of cacti. Discret. Appl. Math. 2016, 203, 217-225. [CrossRef]

30. Palacios, J.L.; Renom, J.M. Another look at the degree-Kirchhoff index. Int. J. Quantum Chem. 2011, 111, 3453-3455. [CrossRef]

31. Shang, Y.L. Further Results on Distance Estrada Index of Random Graphs. Bull. Malaysian Math. Sci. Soc. 2018, 21, 537-544. [CrossRef]

32. Bollobás, B. Modern Graph Theory; Springer: Berlin, Germany, 1998.

33. Feng, L.; Liu, N.; Yu, G.; Li, S. The degree-Kirchhoff index of fully loaded unicyclic graphs and cacti. Util. Math. 2014, 95, 149-159.

34. He, F.; Zhu, Z. Cacti with maximum eccentricity resistance-distance sum. Discret. Appl. Math. 2017, 219, 117-125. [CrossRef]

35. Liu, H.Q.; Lu, M. A Unified Approach to Extremal Cacti for Different Indices. MATCH Commun. Math. Comput. Chem. 2007, 58, 183-194.

(C) 2019 by the authors. Licensee MDPI, Basel, Switzerland. This article is an open access article distributed under the terms and conditions of the Creative Commons Attribution (CC BY) license (http:/ / creativecommons.org/licenses/by/4.0/). 